\title{
Basic study on SH2 domain of Grb2 as a molecular probe for detection of RTK activation
}

\author{
YURIKO SAITO ${ }^{1,3}$, TAKAKO FURUKAWA ${ }^{1,2}$, YASUSHI ARANO ${ }^{3}$, \\ YASUHISA FUJIBAYASHI ${ }^{1,2}$ and TSUNEO SAGA ${ }^{1}$
}

\author{
${ }^{1}$ Molecular Imaging Center, National Institute of Radiological Sciences, 4-9-1, Anagawa, Inage-ku, \\ Chiba 263-8555; ${ }^{2}$ Biomedical Imaging Research Center, University of Fukui, 23-3 Matsuokashimoaizuki, \\ Eiheiji-cho, Yoshida-gun, Fukui 910-1193; ${ }^{3}$ Graduate School of Pharmaceutical Sciences, \\ Chiba University, 1-8-1, Inohana, Chuo-ku, Chiba 260-8675, Japan
}

Received March 16, 2010; Accepted April 23, 2010

DOI: 10.3892/ijo_00000676

\begin{abstract}
Epidermal growth factor receptor (EGFR) and other receptor tyrosine kinases (RTKs) are overexpressed and/or mutated in various cancers and their abnormal activation is implicated in carcinogenesis. We explored the possibility of generating an imaging probe for RTK activation using the $\mathrm{SH} 2$ domain of Grb2 for cancer characterization. For cell penetration, molecular analysis and radiolabeling, the $\mathrm{SH} 2$ domain was fused with TAT, flag and tyrosine residue, respectively (termed TSF). We analyzed TSF characteristics in cells such as cellular uptake, stability and localization. After uptake into EGFR-expressing cells, TSF was found to be binding to phosphorylated-EGFR, which increased by stimulation with EGF. TSF was co-localized with EGFR in EGFR-activated cells, while it was localized as dots in cytosol in EGFR-non-activated cells. Cellular retention time of TSF was significantly extended under EGFR activation with EGF stimuli and reduced under the treatment with a tyrosine kinase inhibitor, Tyrphostin AG1478. In conclusion, the SH2 domain of Grb2 has the potential to be used as a binding component of a probe to detect activated-RTK and to evaluate the effect of kinase inhibitors on RTK activation.
\end{abstract}

\section{Introduction}

Receptor tyrosine kinase (RTK) consists of an extracellular ligand binding domain, a transmembrane domain and an intracellular domain, and is activated by binding with its ligands, typically growth factors. When the ligand binds with its receptor, it induces autophosphorylation of tyrosine (Tyr)

Correspondence to: Dr Takako Furukawa, Molecular Imaging Center, National Institute of Radiological Sciences, 4-9-1, Anagawa, Inage-ku, Chiba 263-8555, Japan

E-mail: tfuru@nirs.go.jp

Key words: receptor tyrosine kinase activation, epidermal growth factor receptor, $\mathrm{SH} 2$ domain, Grb2 residues on the intracellular carboxyl terminal tail of the receptor $(1,2)$. Activated-RTKs can associate with several adaptor proteins containing Src-homology 2 (SH2) domain, such as growth factor receptor bound protein 2 (Grb2), Src homologous and collagen (Shc), and phospholipase $\mathrm{C} \gamma$ (PLC $\gamma$ ). The SH2 domain consists of 100 amino acids and binds to phosphorylated-Tyr of RTKs. The binding leads to the promotion of cell signaling through several pathways to regulate cellular physiology such as cell growth, survival, motility, angiogenesis and differentiation (3-6).

As upregulated expression and activation of RTKs are commonly found in cancers and tightly related to their characteristics (7), RTKs are very attractive targets for cancer diagnosis and therapy (8). There have been various approaches to target RTKs, such as using their ligands, anti-RTK antibodies, and specific Tyr kinase inhibitor (9-16). Most of them target the earlier part of the signaling pathway, that is, from ligand binding to Tyr phosphorylation. However, in some cancers, RTK is known to be mutated or have lost the extracellular ligand binding domain but is activated (7,14,15,17-20). In such cases, the later part of the signaling pathway, that is, from the binding of adaptor proteins to RTKs via the $\mathrm{SH} 2$ domain and thereafter, can provide a desirable target.

In this study, we focused on the $\mathrm{SH} 2$ domain of Grb2 as a key component for designing an imaging probe that would allow the detection of RTK activation. Grb2 is $\sim 25 \mathrm{kDa}$ protein consisting of one SH2 domain flanked by two SH3 domains (21), and intermediates the mitogen-activated protein kinase (MAPK) signaling pathway and also regulates receptor trafficking (22-24). Grb2 works with various RTKs, with epidermal growth factor receptor (EGFR) being its major binding partner $(4,25)$. EGFR, which regulates many cell functions (Fig. 1), is overexpressed and/or mutated in various cancers including breast cancer, head and neck cancer, ovarian cancer and non-small cell lung carcinoma, and has been implicated in the pathogenesis of the disease $(16,19,20)$. In addition, there has been no mutation reported in RTK at the binding sites for the $\mathrm{SH} 2$ domain of Grb2.

Based on these considerations, we designed a protein probe consisting of the SH2 domain of Grb2, HIV-TAT for protein delivery, flag for molecular tag and Tyr residue for 
radiolabeling. We investigated the characteristics of the probe in cells with different activation level of EGFR, as a representative of RTKs, in order to evaluate the possibility to use the probe for in vivo imaging of RTK activation.

\section{Materials and methods}

Cell culture. Human skin cancer A431 cells were obtained from American Type Culture Collection (Manassas, VA, USA) and breast cancer MDA-MB435 cells and lung cancer H3255 cells were kindly provided by Dr R. Nishii of Shiga Medical Center. A431 cells were cultured in Dulbecco's modified Eagle medium (DMEM, Sigma-Aldrich, St. Louis, MO, USA) containing $10 \%$ fetal bovine serum (FBS, SAFC Biosciences, Lenexa, KS, USA), $50 \mathrm{U} / \mathrm{ml}$ of penicillin and $50 \mu \mathrm{g} / \mathrm{ml}$ of streptomycin (Gibco Invitrogen Corp., Carlsbad, CA, USA) in a humidified atmosphere of $5 \% \mathrm{CO}_{2}$ in air at $37^{\circ} \mathrm{C}$. MDAMB435 cells and H3255 cells were cultured in DMEM/F-12 (Sigma-Aldrich), containing 10\% FBS and antibiotics as above.

Plasmids. Grb2 cDNA clone (accession no: NM_203506) was obtained from NIH Mammalian Gene Collection (Invitrogen). The cDNA fragment encoding the SH2 domain fused with TAT was amplified by PCR using a forward primer (5'-ATG TAT GGC AGG AAG AAG CGG AGA CAG CGA CGA AGA GGT GAA ATG AAA CCA CAT CCG TGG-3') and a reverse primer (5'-AAA GAG GGC CTG GAC GTA TGT CGG CTG-3'). Then, for fusion of flag with Tyr sequence, the obtained fragments were amplified by PCR using a forward primer (5'-ATG TAT GGC AGG AAG AAG CG-3') and a reverse primer (5'-TTA GTA CTT ATC GTC GTC ATC CTT GTA ATC GGG CCC AAA GAG GGC CTG G-3'). We also amplified a fragment for control protein without TAT sequence, using a forward primer (5'-ATG GAA ATG AAA CCA CAT CCG TGG-3') and the above reverse primer. To add the matching restriction sites with pGEX-6P-1 or pGEX6P-3 vector (GE Healthcare, Uppsala, Sweden), the fragment was amplified using a forward primer (5'-CCC TGG AGA ATT CAT GTA TGG CAG GAA GAA G-3') or (5'-GAC GGC TTC ATT CCC AAG AAT TCC ATG GAA ATG AAA CCA C-3') and a reverse primer (5'-GCT TAC CGC GGC CGC TTA GTA CTT ATC GTC GTC-3'). The fragments were inserted between EcoRI and NotI sites of the vectors using Ligation High (Toyobo Co., Ltd., Osaka, Japan) to obtain pGEX-TSF and pGEX-SF to express the probe proteins, the SH2 domain fused with TAT, flag and Tyr (TSF), and the SH2 domain fused with flag and Tyr (SF), respectively.

All constructs were transformed into E. coli $\mathrm{DH} 5 \alpha$ (Toyobo) and subsequently screened by restriction analysis, and their DNA sequences were read with ABI PRISM 3100 sequencer (Applied Biosystems, Foster City, CA, USA).

Probe preparation. The GST fusion constructs pGEX-TSF and pGEX-SF were transformed into E. coli BL21 and cultured in Overnight Express Instant TB Medium (Novagen, Darmstadt, Germany) to express GST fusion proteins. Recombinant GST fusion proteins were purified using MicroSpin GST purification module or GSTrap FF. Then, the proteins were digested by PreScission Protease following the manufacturer's

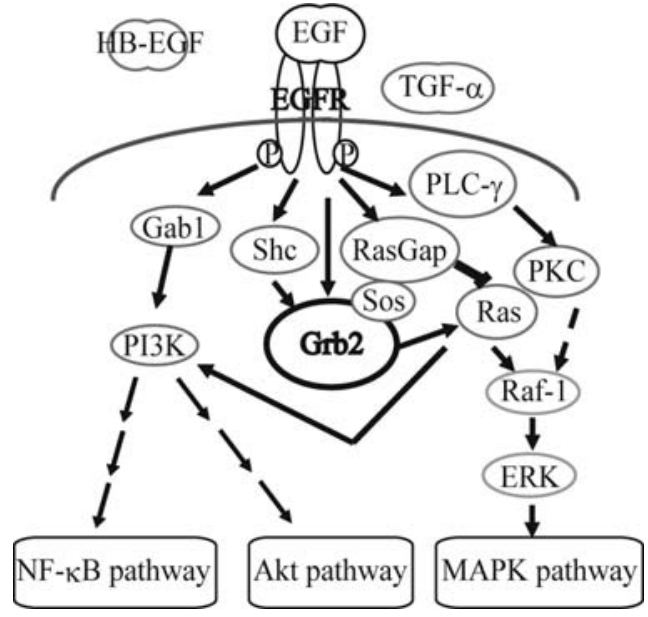

Figure 1. Schematic presentation of EGFR signaling cascade. Grb2 is known to bind to phosphorylated-Tyr ${ }^{1068}$ of EGFR, which accelerates cell growth, survival, motility and differentiation via several pathways.

instructions. Columns and protease were purchased from GE Healthcare.

Probe labeling with ${ }^{125} \mathrm{I}$. ${ }^{125} \mathrm{I}$-labeled protein was prepared by chloramine-T-mediated iodination. Protein $(1 \mu \mathrm{g})$ was mixed with $3.7 \mathrm{MBq}$ of $\mathrm{Na}\left[{ }^{125} \mathrm{I}\right]$ (Perkin Elmer Japan Co., Ltd., Kanagawa, Japan) in $0.3 \mathrm{M}$ phosphate buffer ( $\mathrm{pH} 7.5)$ for $2 \mathrm{~min}$ at room temperature (RT). Chloramine-T ( $1 \mu \mathrm{lof} 4 \mathrm{mg} /$ ml) (Wako Pure Chemical Industries, Ltd., Osaka, Japan) was added to $28 \mu 1$ of the protein mixture and incubated for $5 \mathrm{~min}$ at RT, and the reaction was terminated by adding $6 \mu \mathrm{l}$ of $0.19 \mathrm{mg} / \mathrm{ml}$ sodium metabisulfite (Nacalai Tesque, Kyoto, Japan). The labeled protein was separated from free iodine by gel filteration using a PD10 column (GE Healthcare) equilibrated with $0.1 \mathrm{M}$ acetate buffer $(\mathrm{pH}$ 6.0) containing $1 \%$ bovine serum albumin (Sigma). The labeling efficiency was $\sim 76.8 \pm 6.84 \%$ and an average of 0.8 molecule of ${ }^{125}$ I was calculated to be on one TSF molecule. Column purification resulted in radiochemical purity of $>99 \%$.

Antibodies. Anti-EGFR (R-1), anti-EGFR (1005), and antiactin (C-11) antibodies, donkey anti-goat $\mathrm{IgG}$ conjugated with horseradish peroxidase (HRP), and goat anti-mouse IgG conjugated with HRP were purchased from Santa Cruz Biotechnology (Santa Cruz, CA, USA). Goat anti-rabbit IgG conjugated with HRP was purchased from GE Healthcare. Mouse anti-flag M2 and rabbit anti-flag polyclonal antibodies were from Sigma. Anti-phospho-EGFR $\left(\mathrm{Tyr}^{1068}\right)$ antibody was from Cell Signaling Technology (Beverly, MA, USA). Goat anti-rabbit IgG conjugated with Alexa Flour 488 and goat antimouse IgG conjugated with Alexa Flour 594 were purchased from Invitrogen.

Western blotting. Cell lysates containing 15-20 $\mu \mathrm{g}$ protein or immunoprecipitation samples were subjected to sodium dodecyl sulfate polyacrylamide gel electrophoresis (SDSPAGE) and transferred to PVDF membrane (Millipore, Billerica, MA, USA). They were then probed with primary antibodies. HRP-conjugated secondary antibodies were detected using chemiluminescence with ECL Plus Western 
blotting detection system (GE Healthcare). Bands were visualized by Chemi-Smart 5000 (Vilber Lourmat, Torcy, France) and Chemi-Capt software (Vilber Lourmat). Band intensity was quantified by Image $\mathbf{J}$ software (National Institutes of Health, Bethesda, MD, USA).

Immunoprecipitation. Immunoprecipitation was performed by FLAG Tagged protein immunoprecipitation kit (Sigma). Briefly, total cell lysates containing $\sim 1 \mathrm{mg}$ protein were incubated with washed beads conjugated with anti-flag antibody at $4{ }^{\circ} \mathrm{C}$ overnight. The protein was eluted by $3 \mathrm{X}$ FLAG peptide according to the manufacturer's instructions.

Immunofluorescence staining. Cells were cultured on glass cover slips (Matsunami, Osaka, Japan) and fixed in $4 \%$ paraformaldehyde (Wako) for $20 \mathrm{~min}$ at RT. The cells were then permeabilized with $0.1 \%$ Triton X-100 (Promega KK, Tokyo, Japan) in PBS for 2 min at RT and blocked in 5\% normal goat serum (Sigma) in Blockase (DS Pharma Biomedical, Osaka, Japan) for $30 \mathrm{~min}$ at RT. After the blocking, the cells were incubated with primary antibodies overnight at $4^{\circ} \mathrm{C}$, washed with $0.1 \%$ Tween (Sigma) in PBS (PBST), and then incubated with fluorescence-labeled secondary antibodies for 2-3 $\mathrm{h}$ at RT. After the PBST and PBS washes, the cover slips were mounted with Aqua-Poly/Mount Coverslipping Medium (Polysciences Inc., Warrington, PA, USA). The slides were examined with a confocal fluorescence microscope, FV1000 (Olympus, Tokyo, Japan).

Transduction into cells. For comparison of probe transduction into A431 cells and MDA-MB435 cells, the cells at $4.0 \times 10^{5}$ cells/well in 24 -well plates were incubated with $1.5 \mu \mathrm{M}$ TSF for $1.5 \mathrm{~h}$. After washing the cells with PBS and $0.2 \mathrm{M}$ glycine-HCl buffer ( $\mathrm{pH} 2.0$ ) to remove probes on the cell surface, cell lysates were prepared for Western blotting. The band intensity detected by anti-flag antibody was normalized to actin.

The transduction was also examined with ${ }^{125}$ I-labeled TSF ( $\left.{ }^{125} \mathrm{I}-\mathrm{TSF}\right)$. The cells at $4.0 \times 10^{5}$ cells/well in 24 -well plates were incubated with $1.5 \mathrm{kBq}{ }^{125} \mathrm{I}-\mathrm{TSF}$ for $1.5 \mathrm{~h}$. After washing, the cells were collected and their radioactivity was measured by $\gamma$-counter (Aloka Co., Ltd., Tokyo, Japan).

Probe binding to EGFR. For assessment of the binding of TSF and phosphorylated-EGFR, A431 cells at $3.0 \times 10^{6}$ cells in $100-\mathrm{mm}$ dish were pre-incubated in serum-free media for $24 \mathrm{~h}$, and then treated with $1.5 \mu \mathrm{M}$ TSF or SF in serum-free medium for $1.5 \mathrm{~h}$. EGF at a final concentration of $100 \mathrm{ng} / \mathrm{ml}$ was added to the medium for the last $10 \mathrm{~min}$ of incubation. Cell lysates were prepared for immunoprecipitation to detect TSF-EGFR complex by Western blotting.

Probe retention under EGFR activation. For assessment of the effect of EGFR activation on TSF retention, $4.0 \times 10^{5}$ of serum-starved A431 cells in 24-well plates were incubated with $1.5 \mu \mathrm{M}$ TSF or $1.5 \mathrm{kBq}$ of ${ }^{125} \mathrm{I}-\mathrm{TSF}$ for $1.5 \mathrm{~h}$. After washing, the cells were cultured in serum-free medium with or without $100 \mathrm{ng} / \mathrm{ml} \mathrm{EGF}$ for 0, 10, 30, or $60 \mathrm{~min}$. Cell lysates were prepared at each time point for Western blotting or for the measurement of radioactivity by $\gamma$-counter.
Intracellular localization of the probe under EGFR activation. A431 cells on glass cover slips were cultured in serum-free medium for $24 \mathrm{~h}$, and $1.5 \mu \mathrm{M}$ TSF was added to the cells for $1.5 \mathrm{~h}$. After washing, $100 \mathrm{ng} / \mathrm{ml}$ EGF was added for $10 \mathrm{~min}$. Immunofluorescence staining against TSF and EGFR was performed as described in 'Immunofluorescence staining' using anti-flag and anti-EGFR antibodies as primary antibodies, respectively.

Intracellular localization of the probe and lysosome. A431 cells and MDA-MB435 cells were cultured in serum-added medium on glass cover slips and treated with $1.5 \mu \mathrm{M}$ TSF and $50 \mu \mathrm{M}$ LysoTracker Red (Invitrogen) for $1.5 \mathrm{~h}$. Immunofluorescence staining was performed as described in 'Immunofluorescence staining' to detect TSF and lysosomes.

Comparison of probe retention in A431 cells and MDA-MB435 cells. For comparison of probe stability in A431 cells and MDA-MB435 cells, the cells at $4.0 \times 10^{5}$ cells/well in 24-well plates were incubated with $1.5 \mu \mathrm{M}$ TSF for $1.5 \mathrm{~h}$ in medium containing FBS. After washing, the medium was replaced by the medium without TSF, and the cells were cultured for $30 \mathrm{~min}$. Before and after the $30 \mathrm{~min}$ TSF-free incubation, cell lysates were prepared for Western blotting. The band intensity detected by anti-flag antibody was normalized to actin.

Probe retention under treatment with Tyr kinase inhibitor. $\mathrm{H} 3255$ cells were cultured at $1.0 \times 10^{5}$ cells/well in 24-well plates. Medium was changed to serum-free one and cultured for $48 \mathrm{~h}$. Cells were then incubated with $1.5 \mu \mathrm{M}$ TSF with or without $10 \mu \mathrm{M}$ Tyrphostin AG1478 (Sigma), a highly selective EGFR inhibitor, for $1.5 \mathrm{~h}$. After washing, cells were stimulated with $100 \mathrm{ng} / \mathrm{ml} \mathrm{EGF}$ for $10 \mathrm{~min}$. Cell lysates were prepared for Western blotting. Samples immediately after incubation with TSF, with or without AG1478 treatment, were used as control to evaluate the amount of the intact probe.

Statistical analysis. Aspin-Welch's t-test or Student's t-test was used, depending on the results of F-test.

\section{Results}

Confirmation of purified proteins. For protein delivery into cells, we fused TAT at the $\mathrm{N}$ termini of the $\mathrm{SH} 2$ domain. At $\mathrm{C}$ termini, flag tag and Tyr residue were fused for molecular analysis and radiolabeling, respectively (Fig. 2A). The purified probe proteins, TSF and its TAT-deficient control, termed SF, were identified by SDS-PAGE. Each protein appeared as a single band at the expected molecular weight; TSF was detected at $16 \mathrm{kDa}$ and $\mathrm{SF}$ at $14 \mathrm{kDa}$ (Fig. 2B).

TSF uptake and binding to EGFR. When the probe proteins were incubated with cells in culture, TSF, but not SF, was found in the cells, confirming that the conjugation of TAT enabled the SH2 domain to be delivered into cells (Fig. 3A). We compared TSF uptake into the cells expressing different amount of EGFR, A431 cells overexpressing EGFR and MDA-MB435 cells expressing an undetectable amount of EGFR, by Western blotting. TSF was taken up into both cells, with the amount not significantly different between the two 
A

YGRKKRRQRRRG

EMKPHPWFFGKIPRAKAEEM

LSKQRHDGAFLIRESESAPGD

FSLSVKFGNDVQHFKVLRDG

AGKYFLWVVKFNSLNELVD

YHRSTSVSRNQQIFLRDIEQV

PQQPTYVQALFGP

\section{DYKDDDDKY}

B

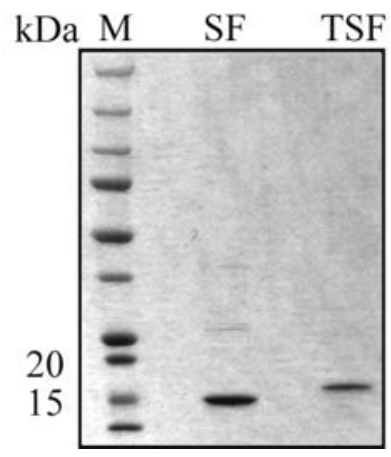

Figure 2. Probe design and confirmation of purified probe proteins. (A) Amino acid sequence of our probe (TSF). TSF consists of 135 amino acid residues containing TAT (black underline) at $\mathrm{N}$ termini of the $\mathrm{SH} 2$ domain and flag (dashed underline) at $\mathrm{C}$ termini. For iodine labeling, Tyr (Y) was added at $\mathrm{C}$ termini of the flag sequence. (B) TSF and SF, control protein without TAT sequence, were identified by SDS-PAGE and CBB staining. TSF was found at $\sim 16 \mathrm{kDa}$ and $\mathrm{SF}$ at $14 \mathrm{kDa}$. The left lane contains the protein marker.

(Fig. 3A). We also compared the transduction efficiency by incubation with ${ }^{125}$ I-TSF and measurement of its radioactivity, and there was no significant difference between the two; it was $24.6 \pm 2.11 \%$ in A431 cells and $20.4 \pm 0.88 \%$ in MDA-MB435 cells (Fig. 3B).

Immunoprecipitation assay confirmed that our probe could bind to phosphorylated-EGFR after the penetration into cells (Fig. 3C). In the absence of EGF, only small amount of phosphorylated-EGFR was found to be binding to TSF. EGF treatment resulted in marked increase in the binding of TSF and phosphorylated-EGFR.

Effect of EGFR activation on TSF retention. To assess whether TSF retention was dependent on EGFR activation, A431 cells cultured in serum-free medium to suppress EGFR activation were treated with TSF for $1.5 \mathrm{~h}$. After the removal of TSF, the cells were cultured in EGF-containing medium to promote EGFR activation. During the first 10 min of EGF treatment, the TSF amount only decreased to $65 \%$, while it rapidly decreased to $37 \%$ in untreated cells. The initial half-life, during which the amount of intact TSF decreased to $50 \%$, was 42 min in EGF-treated cells, while it was 7.2 min in untreated cells (Fig. 4A). The TSF retention was also evaluated with ${ }^{125} \mathrm{I}$-TSF (Fig. 4B). The radioactivity in EGFR-activated cells after the removal of ${ }^{125} \mathrm{I}$-TSF tented to be higher than that in non-activated cells during the period of experiment.
A

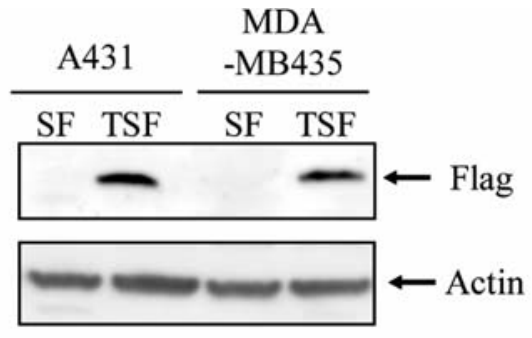

B

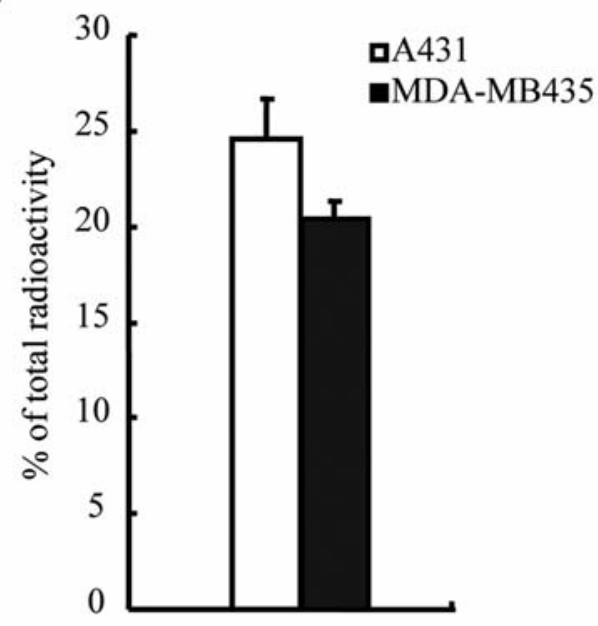

$\mathrm{C}$
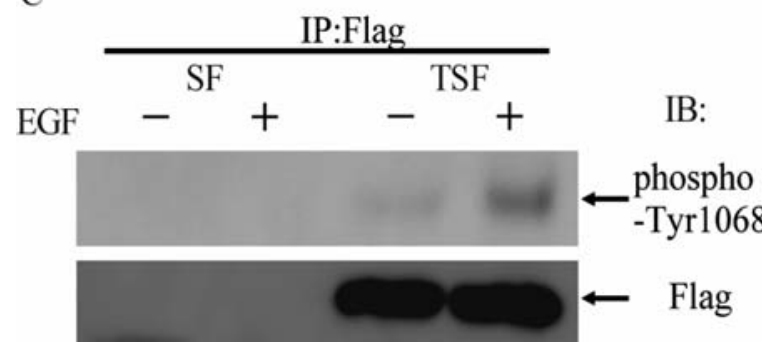

Figure 3. Probe uptake and binding to phosphorylated-EGFR. (A) Cellular uptake of the probes. The probes were incubated with A431 cells (left) and MDA-MB435 cells (right), and the probes in cell lysates were detected by Western blotting. (B) ${ }^{125}$ I-TSF were incubated with A431 and MDA-MB435 cells, and the cellular radioactivity was measured by $\gamma$-counter. The data are expressed as the ratio to total radioactivity, with error bars representing SD $(n=3)$. (C) Probe binding to phosphorylated-EGFR. Cell lysates from TSFor SF-treated A431 cells with or without EGF stimulation were immunoprecipitated by anti-flag antibody and detected with anti-phosphorylated$\mathrm{Tyr}^{1068}$ of EGFR (upper panel) and anti-flag (lower panel) antibodies.

Effect of EGFR activation on TSF localization in cells. Fig. 5 shows the immunofluorescence staining examining the TSF distribution in A431 cells under EGFR activation. TSF co-localized with EGFR near the cell membrane in EGFRactivated cells. On the other hand, it was localized as dots in cytosol in EGFR-non-activated cells, and the fluorescence intensity of TSF was seemed to be lower than that in EGFRactivated cells.

Comparison of intracellular localization of TSF, lysosome and TSF retention. Since TSF seemed to be susceptible to protein degradation in Western blotting study, we examined the subcellular localization of TSF with lysosomes where endocytosed proteins are known to be degraded (Fig. 6A). In A431 cells, some TSF was co-localized with lysosomes, but most 
A
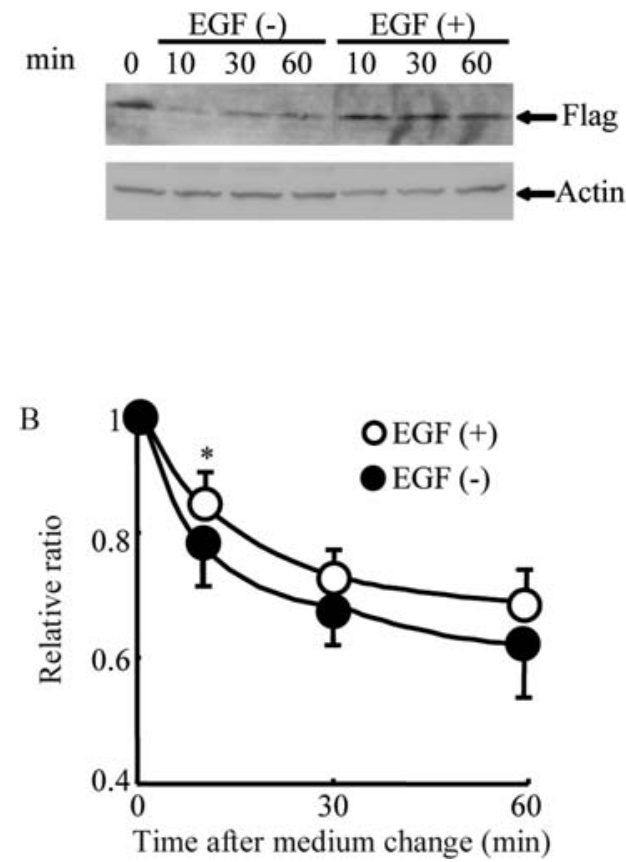

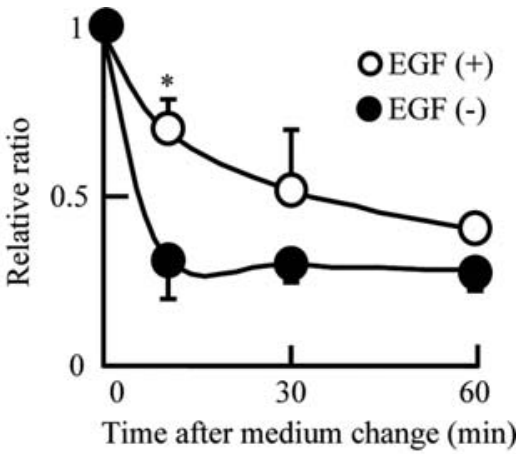

Figure 4. Effect of EGFR activation on TSF retention. (A) The left panel shows the result of Western blotting with anti-flag (upper panel) and antiactin (lower panel) antibodies after incubation of A431 cells with TSF. The right graph shows the relative change of intact TSF. The band intensity of TSF was normalized to actin, and expressed as the ratio to that at time 0 , with error bars representing SD (n=3). ${ }^{*} \mathrm{p}<0.05$ between EGF (+) and EGF (-). (B) After incubation with ${ }^{125} \mathrm{I}-\mathrm{TSF}$ and its removal from the media, the cells were measured for radioactivity at indicated time points. Data are expressed as the ratio to that at time 0 , immediately after the removal of ${ }^{125} \mathrm{I}$-TSF, with error bars representing SD (n=3). ${ }^{*} \mathrm{p}<0.05$ between EGF (+) and EGF (-).

A
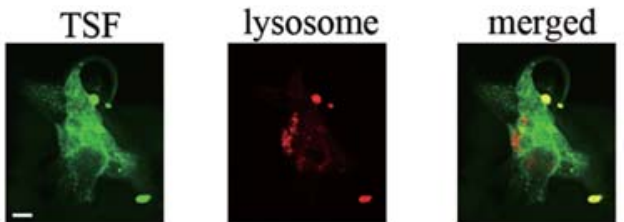

MDA-MB435
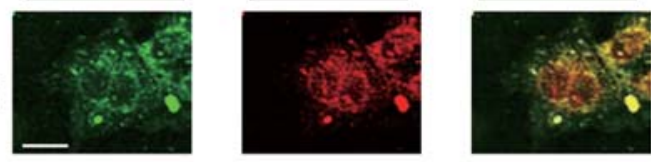

B

Figure 5. Effect of EGFR activation on TSF localization. Immunofluorescence staining in EGF-untreated (upper panels) and EGF-treated A431 cells (lower panels). The cells were stained with anti-flag (green) and anti-EGFR (red) antibodies. Yellow indicates overlapped staining. Scale bar indicates $20 \mu \mathrm{m}$ in each image.

was found near the cell membrane, and did not overlap with lysosomes. On the other hand, in MDA-MB435 cells, TSF and lysosomes were mostly co-localized as dots in cytosol. When comparing the intact TSF remaining in cells at $30 \mathrm{~min}$ after the removal of TSF, the amount of TSF in MDA-MB435 cells was significantly less than that in A431 cells (Fig. 6B).

Effect of Tyr kinase inhibitor on TSF retention. We also examined the TSF retention under EGFR inactivation by treatment with a chemical inhibitor (Fig. 7). H3255 cells are known to overexpress EGFR with L858R mutation and be sensitive to Tyr kinase inhibitors. In H3255 cells, EGFR was phosphorylated even in the absence of any added growth factor and when the cells were treated with AG1478, a specific EGFR inhibitor, the phosphorylated-Tyr ${ }^{1068}$ of EGFR was decreased regardless of EGF treatment. The transduction efficiency was not affected by AG1478 treatment. At $10 \mathrm{~min}$ after the removal of TSF from medium, TSF amount in

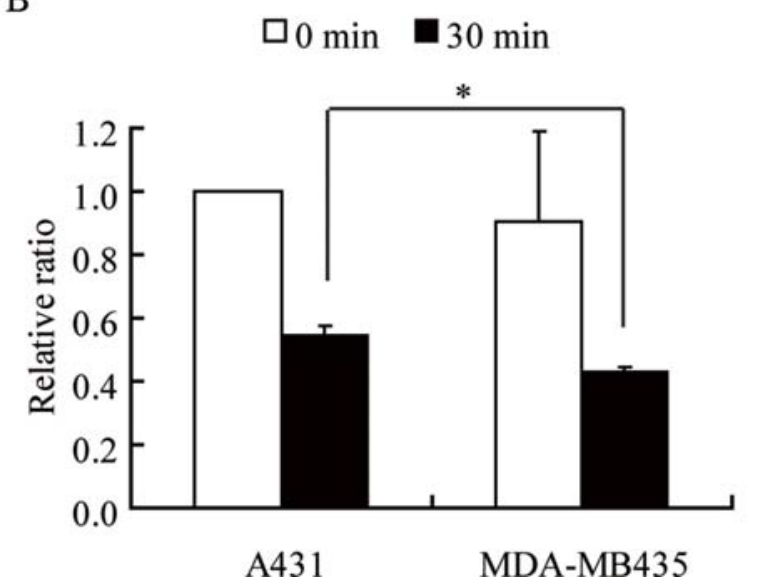

Figure 6. Intracellular localization of TSF and TSF retention in cells expressing different amount of EGFR. (A) Double staining for TSF and lysosome. A431 cells (upper panels) and MDA-MB435 cells (lower panels) were treated with TSF and LysoTracker Red (red), and stained with anti-flag (green) antibody. Yellow indicates overlapped staining. Scale bar indicates $20 \mu \mathrm{m}$ in each image. (B) TSF retention in A431 cells and MDA-MB435 cells. The cells were treated with TSF and then incubated in TSF-free medium for $30 \mathrm{~min}$. TSF in the cell lysates before and after the TSF-free incubation were subjected to Western blotting. The band intensity detected by anti-flag antibody was normalized to actin and the ratio to that in A431 cells before the incubation was calculated. ${ }^{*} \mathrm{p}<0.05$ between A431 cells and MDA-MB435 cells. 


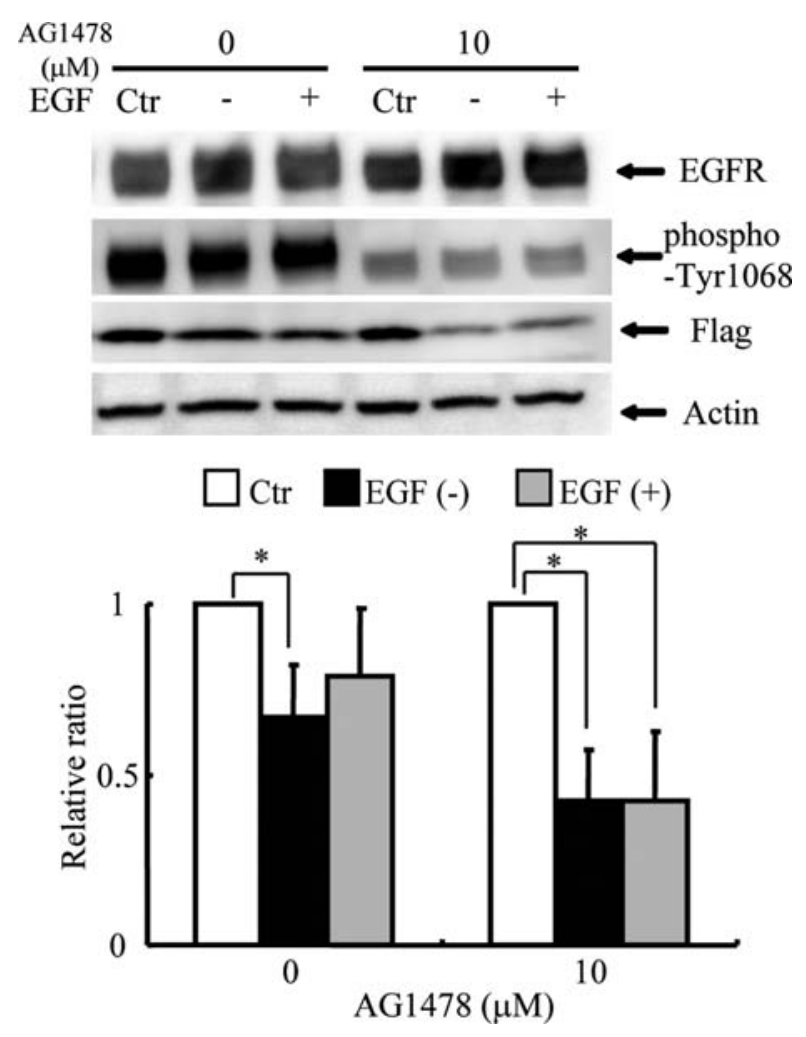

Figure 7. Effect of tyrosine kinase inhibitor on TSF retention. H3255 cells in serum-free medium were pre-treated with TSF, with or without $10 \mu \mathrm{M}$ AG1478, and then stimulated with EGF. The upper panel shows the result of Western blotting and the lower graph shows the relative change of intact TSF. Ctr represents the cells immediately after TSF treatment with or without AG1478, and used as control in each treatment. ${ }^{*} \mathrm{p}<0.05(n=3)$ between each Ctr and EGF (-) or EGF (+).

AG1478-treated cells was lower than that in untreated cells. The cells immediately after TSF treatment with or without AG1478 were used as control in each treatment (Ctr), and the ratio to Ctr was $0.43 \pm 0.15$ in EGF (-) and $0.42 \pm 0.20$ in EGF $(+)$ in AG1478-treated cells, while $0.67 \pm 0.15$ in EGF (-) and $0.79 \pm 0.20$ in EGF $(+)$ in untreated cells.

\section{Discussion}

There have been studies proposing the utilization of the $\mathrm{SH} 2$ domain of the binding partners of membrane RTKs for treatment of cancer through inhibition of signal transduction, imaging and determination of its function $(13,26)$. In the present study, we designed a novel molecular probe containing the $\mathrm{SH} 2$ domain of Grb2 and examined its properties in cells to assess the possibility of its future use for in vivo imaging of RTK activation, selecting EGFR as its target molecule for the first step. We envisaged that the signal activation would be detected by the different probe retention in cells with presence or absence of phosphorylated-RTK. For protein transduction into cells, we selected HIV-TAT sequence. TAT is one of the cell-penetrating peptides (CPPs) and can deliver protein of $>120 \mathrm{kDa}$ into living cells and mouse tissues $(11,27)$. It is also reported that protein delivery by TAT is less cytotoxic than that of other CPPs such as polyarginine and transportan $(12,28)$. Our probe, TSF, was transduced into cells with both high and low EGFR expression with similar efficiency. This seemed reasonable, as protein transduction by CPP is mostly dependent on the characteristics of the cell membrane and cell surface area for attachment, and the early uptake stage could depend on the characteristics of CPP and not on the expression or activation of EGFR.

To distinguish the cells with different levels of RTK activation by protein probe accumulation, it is necessary to understand and exploit protein metabolism or trafficking after its penetration into the cells. Our probe was unstable in cells and intact probe protein was decreased to $\sim 40 \%$ in $1 \mathrm{~h}$ even in EGFR-activated cells. Fischer et al reported that TAT labeled with fluorescence showed much lower uptake into cells than other CPPs such as polyarginine and antennapedia because TAT fusion protein was susceptible to intracellular protease and quickly exited from cells (29). This study supports the concept that TSF instability might be due to TAT characteristics.

In this study, extended retention of TSF was observed in EGFR-activation study. In our preliminary experiment, EGFR was highly phosphorylated at 10-30 min after EGF stimulation, matching the time frame when the extended retention was observed. Furthermore, treatment with EGFR kinase inhibitor resulted in the decrease of $\mathrm{Tyr}^{1068}$ phosphorylation and shortened TSF retention. Combined with the results of immunoprecipitation and immunofluorescence staining experiments, it could be assumed that EGFR activation could stabilize TSF by an increase in the binding with phosphorylated-EGFR located at the cell membrane. Although the TAT transduction mechanism is still controversial $(30,31)$, TAT fusion protein is thought to penetrate into cells in a charge-dependent manner and be brought into endosome (32). When A431 cells under the normal culture condition were incubated with TSF and lysosome marker, most of TSF showed different distribution from lysosome while they were co-localized in MDA-MB435 cells, which showed shorter retention of TSF than in A431 cells. This also indicates that the stabilization of TSF would be due to the evacuation from protein degradation.

In this study, flag tag was used for most of the experiments to understand and evaluate the basic characteristics of the probe. For in vivo imaging, the probe should be labeled with a 'beacon' detectable from outside of living cells or bodies. Fluorescent dyes would be suitable for imaging at cellular level and radioisotopes for PET (positron emission tomography) or SPECT (single photon emission computed tomography) imaging at whole-body level. Cellular retention of ${ }^{125}$ I-TSF, as observed by radioactivity, was higher in EGFRactivated cells than in control cells, suggesting the possibility that radio-iodinated TSF can capture the EGFR activation. ${ }^{125}$ I-labeling can be easily replaced by ${ }^{124}$ I-labeling. ${ }^{124} \mathrm{I}$ is a positron emitter widely used for PET imaging both in animal and human studies $(33,34)$. However, the less prominent difference between EGFR-activated cells and non-activated cells observed by radioactivity than by Western blotting, which might be attributable to partially degraded TSF with radiolabel, indicates that more suitable labeling methods and radionuclides should be explored.

As Grb2 also binds to phosphorylated-Tyr containing sequences of RTKs other than EGFR, it is likely that TSF 
would also be able to bind to those phosphorylated-receptors and the behavior of TSF, such as cellular retention and localization, would be influenced by the activation of those receptors. The importance of Grb2 signaling in cell cycle progression and cell motility is now increasingly recognized (35). Although only the activation of EGFR was examined in this study, it would be interesting to explore the possibility to capture the activation of other signaling pathways going through Grb2 using TSF.

In conclusion, we showed that the stability of the $\mathrm{SH} 2$ domain of Grb2 was dependent on the cellular level of EGFR activation in EGFR expressing cells. We hypothesized the trafficking of our probe as follows: TSF could leave endosome relatively quickly into cytosol and bind to phosphorylatedEGFR; if there is no binding partner, TSF will be degraded by proteases in cytosol or in lysosome. Our probe still requires improvements, however, the $\mathrm{SH} 2$ domain of Grb2 can be used as a binding component of molecular probes for detecting activated-RTK in cancer and also for evaluating the effect of kinase inhibitors on RTK activation.

\section{Acknowledgements}

We would like to thank the members of the Diagnostic Imaging Group, Molecular Imaging Center, NIRS, for helpful discussions and valuable suggestions. We would also like to thank Ms. C. Sogawa for her help with the animal experiments, Mr. A. Gerz for English correction, and Ms. R. Miyamoto for advice on the experimental procedures. This study was supported by grants from the Japan Society for the Promotion of Sciences (Grant no.: 20790921) and the National Institute of Radiological Sciences.

\section{References}

1. Ullrich A and Schlessinger J: Signal transduction by receptors with tyrosine kinase activity. Cell 61: 203-212, 1990.

2. Kaushansky A, Gordus A, Chang B, Rush J and MacBeath G: A quantitative study of the recruitment potential of all intracellular tyrosine residues on EGFR, FGFR1 and IGF1R. Mol Biosyst 4 643-653, 2008

3. Sundaram MV: RTK/Ras/MAPK signaling. WormBook 1-19, 2006.

4. Schulze WX, Deng L and Mann M: Phosphotyrosine interactome of the ErbB-receptor kinase family. Mol Syst Biol 1: 2005.0008, 2005.

5. Machida K and Mayer BJ: The SH2 domain: Versatile signaling module and pharmaceutical target. Biochim Biophys Acta 1747: $1-25,2005$

6. Blume-Jensen P and Hunter T: Oncogenic kinase signalling. Nature 411: 355-365, 2001.

7. Robertson SC, Tynan JA and Donoghue DJ: RTK mutations and human syndromeswhen good receptors turn bad. Trends Genet 16: 265-271, 2000.

8. Zwick E, Bange J and Ullrich A: Receptor tyrosine kinase signalling as a target for cancer intervention strategies. Endocr Relat Cancer 8: 161-173, 2001.

9. Niu G and Chen X: PET imaging of angiogenesis. PET Clin 4: $17-38,2009$

10. Snyder EL, Saenz CC, Denicourt C, et al: Enhanced targeting and killing of tumor cells expressing the CXC chemokine receptor 4 by transducible anticancer peptides. Cancer Res 65 : 10646-10650, 2005.

11. Kim D, Jeon C, Kim JH, et al: Cytoplasmic transduction peptide (CTP): New approach for the delivery of biomolecules into cytoplasm in vitro and in vivo. Exp Cell Res 312: 1277-1288, 2006.

12. Jones SW, Christison R, Bundell K, et al: Characterisation of cell-penetrating peptide-mediated peptide delivery. Br J Pharmacol 145: 1093-1102, 2005.
13. Katterle Y, Brandt BH, Dowdy SF, Niggemann B, Zanker KS and Dittmar T: Antitumour effects of PLC-gamma1-(SH2)2TAT fusion proteins on EGFR/c-ErbB-2-positive breast cancer cells. Br J Cancer 90: 230-235, 2004.

14. Janne PA, Engelman JA and Johnson BE: Epidermal growth factor receptor mutations in non-small-cell lung cancer: Implications for treatment and tumor biology. J Clin Oncol 23: 3227-3234, 2005

15. Greulich H, Chen TH, Feng W, et al: Oncogenic transformation by inhibitor-sensitive and -resistant EGFR mutants. PLoS Med 2: E313, 2005.

16. Wikstrand CJ, Hale LP, Batra SK, et al: Monoclonal antibodies against EGFRvIII are tumor specific and react with breast and lung carcinomas and malignant gliomas. Cancer Res 55: 3140-3148, 1995.

17. Graveel CR, DeGroot JD, Su Y, et al: Met induces diverse mammary carcinomas in mice and is associated with human basal breast cancer. Proc Natl Acad Sci USA 106: 12909-12914, 2009.

18. Ostrowski J, Polkowski M, Paziewska A, et al: Functional features of gene expression profiles differentiating gastrointestinal stromal tumours according to kit mutations and expression. BMC Cancer 9: 413, 2009.

19. Kobayashi S, Boggon TJ, Dayaram T, et al: EGFR mutation and resistance of non-small-cell lung cancer to gefitinib. N Engl J Med 352: 786-792, 2005.

20. Chu CT, Everiss KD, Wikstrand CJ, Batra SK, Kung HJ and Bigner DD: Receptor dimerization is not a factor in the signalling activity of a transforming variant epidermal growth factor receptor (EGFRvIII). Biochem J 324: 855-861, 1997.

21. Matuoka K, Shibata M, Yamakawa A and Takenawa T: Cloning of ASH, a ubiquitous protein composed of one Src homology region $(\mathrm{SH}) 2$ and two $\mathrm{SH} 3$ domains, from human and rat cDNA libraries. Proc Natl Acad Sci USA 89: 9015-9019, 1992.

22. Burke P, Schooler K and Wiley HS: Regulation of epidermal growth factor receptor signaling by endocytosis and intracellular trafficking. Mol Biol Cell 12: 1897-1910, 2001.

23. Yamazaki T, Zaal K, Hailey D, Presley J, Lippincott-Schwartz J and Samelson LE: Role of Grb2 in EGF-stimulated EGFR internalization. J Cell Sci 115: 1791-1802, 2002.

24. Jiang X, Huang F, Marusyk A and Sorkin A: Grb2 regulates internalization of EGF receptors through clathrin-coated pits. Mol Biol Cell 14: 858-870, 2003.

25. Seiden-Long I, Navab R, Shih W, et al: Gab1 but not Grb2 mediates tumor progression in Met overexpressing colorectal cancer cells. Carcinogenesis 29: 647-655, 2008.

26. Li W, Li F, Huang Q, Frederick B, Bao S and Li CY: Noninvasive imaging and quantification of epidermal growth factor receptor kinase activation in vivo. Cancer Res 68: 4990-4997, 2008.

27. Schwarze SR, Ho A, Vocero-Akbani A and Dowdy SF: In vivo protein transduction: Delivery of a biologically active protein into the mouse. Science 285: 1569-1572, 1999.

28. Jiang T, Olson ES, Nguyen QT, Roy M, Jennings PA and Tsien RY: Tumor imaging by means of proteolytic activation of cell-penetrating peptides. Proc Natl Acad Sci USA 101: $17867-17872,2004$.

29. Fischer R, Kohler K, Fotin-Mleczek M and Brock R: A stepwise dissection of the intracellular fate of cationic cell-penetrating peptides. J Biol Chem 279: 12625-12635, 2004.

30. Prochiantz A: Messenger proteins: Homeoproteins, TAT and others. Curr Opin Cell Biol 12: 400-406, 2000.

31. Tunnemann G, Martin RM, Haupt S, Patsch C, Edenhofer F and Cardoso MC: Cargo-dependent mode of uptake and bioavailability of TAT-containing proteins and peptides in living cells. FASEB J 20: 1775-1784, 2006 .

32. Fittipaldi A, Ferrari A, Zoppe M, et al: Cell membrane lipid rafts mediate caveolar endocytosis of HIV-1 Tat fusion proteins. J Biol Chem 278: 34141-34149, 2003.

33. Sundaresan G, Yazaki PJ, Shively JE, et al: ${ }^{124} \mathrm{I}$-labeled engineered anti-CEA minibodies and diabodies allow highcontrast, antigen-specific small-animal PET imaging of xenografts in athymic mice. J Nucl Med 44: 1962-1969, 2003.

34. Phan HT, Jager PL, Paans AM, et al: The diagnostic value of ${ }^{124} \mathrm{I}-\mathrm{PET}$ in patients with differentiated thyroid cancer. Eur J Nucl Med Mol Imaging 35: 958-965, 2008.

35. Giubellino A, Burke TR Jr and Bottaro DP: Grb2 signaling in cell motility and cancer. Expert Opin Ther Targets 12: 1021-1033, 2008. 